\title{
ESTUDIO COMPARADO: LA EDUCACIÓN FÍSICA EN COLOMBIA, CHILE, ESPAÑA, PORTUGAL, REPÚBLICA DOMINICANA Y VENEZUELA
}

\author{
Comparative study: Physical Education in Colombia, \\ Chile, Spain, Portugal, Dominican Republic and Venezuela
}

(iD) Miguel Ángel González-Valeiro*1

miguel.gonzalez.valeiro@udc.es

Sirley Andrea Bustamante-Castaño

sirley.bustamante@udea.edu.co

(iD) Beatriz Chaverra-Fernández ${ }^{2}$

beatriz.chaverra@udea.edu.co

iD Lúcia Cristina da Fonseca-Gomes ${ }^{3}$

luciagomes12@gmail.com

iD Rosa López-D’Amico ${ }^{4}$

rlopezdedamico@yahoo.com

(iD) João Filipe da Silva Figueira-Martins ${ }^{5}$ jmartins@fmh.ulisboa.pt

iD Alda Marysol Reyno-Freundt ${ }^{6}$ aldareyno@gmail.com

iD Belén Toja-Reboredo ${ }^{7}$ belen.toja@udc.es

Henyer Ramón Zamora-Mota ${ }^{8}$

henyer.zamora@isfodosu.edu.do

* Autor por correspondencia

${ }^{1}$ Universidade da Coruña, España

${ }^{2}$ Instituto Universitario de Educación Física de la Universidad de Antioquia, Colombia

${ }^{3}$ Universidade Lusófona de Humanidades e

Tecnologias, Portugal

${ }^{4}$ Universidad Pedagógica Experimental Libertador -Maracay, Venezuela

${ }^{5}$ Universidade de Lisboa, Lisboa, Portugal

${ }^{6}$ Universidad Metropolitana de Ciencias de la Educación - Universidad de Playa Ancha, Chile

${ }^{7}$ Universidade da Coruña, España

${ }^{8}$ Instituto Superior de Formación Docente

Salomé Ureña, República Dominicana

Fecha recepción: 15/8/2019

Fecha aprobación: 10/11/2019

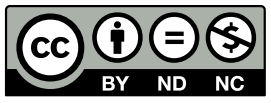
Esta obra está bajo la licencia de Creative
Commons Reconocimiento-Nocomercial-SinobraDerivada 4.0 intermacional.

ISSN (impreso): 2636-2139

ISSN (en línea): 2636-2147

Sitio web: https://revistas.isfodosu.edu.do/recie

\section{Resumen}

En este artículo se presenta un estudio comparado de la Educación Física en lo relativo a la legislación, su estructura, el currículo, las escuelas y el profesorado, todo ello en países dentro del marco que compone la Red Euroamericana de Actividad Física, Educación y Salud (REAFES), con la característica de ser todos iberoamericanos y no anglófonos. Para ello, se elaboró un cuestionario de respuestas abiertas siguiendo los indicadores para una enseñanza de calidad para la Educación Física (EF) establecidos por la UNESCO a partir del análisis de los resultados de la aplicación de la Encuesta Mundial sobre la situación de la Educación Física (North Western Countries Physical Education Association, 2014). Se envía a expertos en el ámbito de los países implicados, recibiendo la respuesta de Colombia, Chile, España, Portugal, República Dominicana y Venezuela. Se analizan las encuestas comparando cada variable en cada uno de los países para poder obtener un abordaje de mayor profundidad que si analizáramos país por país. Se destaca la idea de que en todos los países analizados está garantizado (excepto excepciones coyunturales dentro de cada uno), una estructura, un currículo y una cualificación docente que asegura de forma suficiente los mínimos requeridos para el desarrollo de una Educación Física de calidad y, por el contrario, es a la hora de establecer programas fuera del horario lectivo (educación formal), donde se encuentran mayores diferencias en las intenciones y riquezas de los programas de actividades fuera de estos horarios (educación informal y no formal).

Palabras clave: calidad, currículo, Educación Física, estudio comparativo, formación de docentes, formador de profesores.

\section{Abstract}

This article presents a comparative study of Physical Education in terms of legislation, structure, curriculum, schools and teachers, in the countries within the framework of the Euro-American Network on Physical Activity, Education and Health (REAFES) with the characteristic of being Latin American countries and not Anglophone. An open-ended questionnaire is prepared following the indicators for quality education for Physical Education established by UNESCO based on the analysis of the results of the implementation of the "Global Survey on the Situation of Physical Education" (North Western Countries Physical Education Association, 2014). It is sent to experts in the field and answers are received from Colombia, Chile, Spain, Portugal, Dominican Republic and Venezuela. Surveys are analyzed by comparing each variable in each of the countries in order to obtain a deeper approach than if analyzed country by country. We emphasize the idea that in all the countries analyzed a structure, a curriculum and a teaching qualification are guaranteed (except for short-term cases within each one) that sufficiently ensures the minimum required for the development of quality physical education and, on the contrary, it is when establishing programs outside school hours (formal education), where there are greater differences in the intentions and wealth of activity programs outside these hours (informal and non-formal education).

Keywords: curriculum, comparative study, quality, Physical Education; teacher training. 


\section{Introducción}

Si bien hay estudios publicados sobre Educación Física comparada, como los de Pühse y Gerber (2005), Klein y Hardman (2008), Ribeiro et al. (2010), Chin y Edginton (2014), Aguilar-Farias et al. (2018), y la Organización para la Cooperación y el Desarrollo Económico (OECD, 2019), así como la existencia de una asociación internacional orientada a este tipo de temática, la International Society for Comparative Physical Education and Sport, que dispone de una revista de publicación periódica, el Journal of International Society for Comparative Physical Education and Sport, en este trabajo se presentan algunas similitudes y diferencias, en los modelos educativos a nivel legislativo, en la implementación de esta materia en el currículo escolar y de las condiciones formativas y profesionales de los docentes en aquellos países que se encuentran en la órbita de las actividades de formación permanente que, viene organizando y/o participando el Instituto Superior de Formación Docente Salomé Ureña(cursos de postgrado másteres universitarios, doctorado y congresos, entre otros), y en la colaboración de éste centro con la Red Euroamericana de Actividad Física, Educación y Salud (REAFES).

\section{Análisis de la literatura}

Los estudios comparados en este ámbito cubren un gran número de países. Pühse y Gerber (2005), aborda 35 países de los cinco continentes, tratando temas más centrados en la EF escolar a nivel curricular. En tanto que, Klein y Hardman (2008), realizó en un elevado número de países europeos, analizando aspectos relativos al marco geográfico, educativo, político e institucional, la organización de la EF y la Educación Deportiva, la existencia de actividades extracurriculares y/o extraescolares y los programas de EF. Ribeiro et al. (2010) se centra en los resultados de la revisión sistemática del proyecto Guide for Useful Interventions for Activity in Latin America, relacionado con los programas de EF, en las escuelas de América Latina. Los objetivos describieron cinco programas de EF (un estudio en Brasil, dos en Chile y dos en la frontera entre Estados
Unidos y México), discutir las implicaciones para las recomendaciones efectivas de EF en la escuela, proponer enfoques para implementar estas intervenciones e identificar lagunas en la literatura de la investigación relacionada con la promoción de la actividad física en América Latina en jóvenes. En el año 2014, Chin y Edginton, abordaron aspectos en torno a la práctica de la actividad física y programas escolares y comunitarios orientados a la salud (obesidad, estilos de vida, etc.), en 40 países de los cinco continentes. Y, por último, el estudio de Aguilar-Farias et al. (2018), se basa en 26 países de América y el Caribe, pretendiendo describir y comparar la actividad física en general, el transporte activo hacia y desde la escuela, la participación en EF y el comportamiento sedentario.

Por otro lado, la Organización de las Naciones Unidas para la Educación, la Ciencia y la Cultura (UNESCO), publica en 2015 el documento titulado Educación Física de calidad: guía para los responsables politicos (McLennan \& Thompson, 2015), en que se insta a los gobiernos y a los planificadores de los sistemas educativos a invertir la tendencia negativa existente en la práctica de actividad física, que la Organización Mundial de la Salud (OMS) ha calificado de verdadera epidemia, ya que cada año causa la muerte de unos 3.2 millones de personas, cifra dos veces mayor que las defunciones ocasionadas por el sida en nuestro planeta. Esta guía trata de abordar siete cuestiones preocupantes que puso de manifiesto la Encuesta Mundial sobre la situación de la EF (North Western Countries Physical Education Association, 2014) llevada a cabo por la UNESCO: (1) La diferencia persistente que se da entre las políticas de EF adoptadas y su aplicación práctica. (2) Las continuas deficiencias observadas en la asignación de horarios para la EF en los currículos escolares. (3) Los problemas relacionados con la pertinencia y calidad del currículo de EF en los programas educativos. (4) La insuficiente calidad de los programas de formación inicial para docentes. (5) La calidad y el mantenimiento deficientes de las instalaciones para actividades físicas y deportivas. (6) La persistencia de los obstáculos que impiden impartir una EF por igual a todos los escolares, así como el acceso de todos ellos a la misma. (7) La coordinación 
insuficiente de los componentes de las comunidades de padres y docentes.

En el documento anteriormente mencionado de la UNESCO, se establecen unos indicadores de calidad, en concreto: (1) Cumplir las necesidades básicas, que comprende como indicadores: política (legislación), recursos humanos, recursos materiales, capacitación, promoción y seguimiento y evaluación; (2) Proporcionar una EF de calidad: currículo, enlaces transversales/externos, estudiantes, evaluación e investigación; (3) Garantizar la formación de profesores de EF de calidad: perfil profesional, conocimientos, aptitud para la docencia, práctica docente, cualificación del curso y seguimiento y evaluación.

Finalmente, la OCDE (2019) presenta: (1) la perspectiva internacional sobre las características clave de la EF y la educación para salud en clave de regulación legislativa y centrada específicamente en el diseño curricular; (2) la recogida de evidencias científicas sobre la asociación entre EF y actividad física y estilos de vida activos y saludables (físicos-psicológicos-afectivos-cognitivos-sociales) en niños y jóvenes, y si los países participantes integran estos conocimientos en su currículo y, por último, (3) un análisis comparativo sobre las líneas futuras que seguirán los currículos de la EF orientada a la salud, revisando a la vez las medidas que se están implementando en algunos países, lo que permite tener una visión prospectiva de las posibles medidas a implementar.

A la luz de los anteriores antecedentes y atendiendo a los indicadores de calidad mencionados, este estudio tiene como objetivo realizar una comparación entre los países sobre el estado de la EF como materia del currículo escolar, la formación del profesorado del área y el estatus profesional en relación con los de otras materias del currículo.

\section{Método}

Se elaboró un cuestionario de respuestas abiertas que fue remitido a un grupo de expertos en el ámbito de la formación de profesores de EF. Revisado el mismo y recogidas sus sugerencias, se modificó el original y se remitió la versión definitiva (Anexo 1) a un grupo de docentes universitarios que participan en centros de formación superior en cada uno de los países intervinientes en este artículo, solicitándoles que lo respondieran. Se envió a profesores de siete países (Chile, Colombia, España, México, Portugal, República Dominicana y Venezuela; se recibió respuesta de seis de ellos (excepto México).

El cuestionario está organizado en seis grandes dimensiones, cada una con diferentes variables, como puede verse en la Tabla 1.

Tabla 1. Dimensiones y variables del cuestionario

\begin{tabular}{|c|c|}
\hline Dimensiones & Variables \\
\hline $\begin{array}{l}\text { Legislación de la } \\
\text { Educación en el } \\
\text { país }\end{array}$ & $\begin{array}{l}\text { a.- Regulación legislativa } \\
\text { b.- Nivel educativo } \\
\text { c.- Tipo de escuela: públicas, priva- } \\
\text { das, subvencionadas o concer- } \\
\text { tadas }\end{array}$ \\
\hline $\begin{array}{l}\text { La EF en el currí- } \\
\text { culo nacional y el } \\
\text { deporte escolar }\end{array}$ & $\begin{array}{l}\text { a.- Número de horas semanales de } \\
\text { EF en la enseñanza primaria } \\
\text { b.- Número de horas semanales de } \\
\text { EF en la enseñanza secundaria }\end{array}$ \\
\hline $\begin{array}{l}\text { La EF en el currícu- } \\
\text { lo escolar }\end{array}$ & $\begin{array}{l}\text { a.- El currículo de la EF en la legis- } \\
\text { lación: grado de prescripción y } \\
\text { carácter (nacional/estadal/pro- } \\
\text { vincial/municipal } \\
\text { b.- Elementos didácticos recogidos } \\
\text { en dicha legislación } \\
\text { c.- Existencia de programas de de- } \\
\text { porte escolar } \\
\text { (funcionamiento y coordinación) }\end{array}$ \\
\hline Las escuelas & $\begin{array}{l}\text { a. Calidad de material e instala- } \\
\text { ciones } \\
\text { b. Existencia de actividades ex- } \\
\text { traescolares } \\
\text { c. Práctica de deporte de club en } \\
\text { las instalaciones de las escuelas }\end{array}$ \\
\hline El profesorado & $\begin{array}{l}\text { a. Formación inicial del profeso- } \\
\text { rado } \\
\text { b. Formación continua del profe- } \\
\text { sorado } \\
\text { c. Sistemas de acceso al cuerpo } \\
\text { docente de las escuelas públi- } \\
\text { cas } \\
\text { d. Condiciones laborales del profe- } \\
\text { sor de EF }\end{array}$ \\
\hline
\end{tabular}

Recibidos los cuestionarios, se realizó un análisis de su contenido semántico, trabajando de forma induc- 
tiva a partir de las respuestas recibidas en cada una de las dimensiones/variables, clasificando las respuestas en virtud de su naturaleza.

Una vez elaborado un primer borrador, se reenvió a cada uno de los expertos encuestados para que comprobaran que el texto se ajustara a la realidad de cada país; realizando en paralelo una validación y un ajuste de la interpretación. Tras otra revisión, se envió la nueva versión de los resultados para que confirmaran su acuerdo.

\section{Resultados}

A continuación se analizan los resultados, a partir de las dimensiones en las cuales se estructuró el cuestionario (Tabla 1); realizando previamente una enumeración de las leyes de educación, que son aplicables a cada uno de los países objeto de este estudio, para poder interpretar algunas de las comparaciones que se van a presentar (Ley General de Educación 20370/2009. Ministerio de Educación. Gobierno de Chile, 2009, Ley No 20.529/2011, 2011; Ley General de Educación 115/1994. Congreso de la República de Colombia, 1994; Gobierno de España, 2013; Lei de Bases do Sistema Educativo46/1986. Assembleia da República de Portugal, 1986; Ley Orgánica de Educación de la República Dominicana 66-1997. Congreso Nacional de la República Dominicana, 1997; Ley Orgánica de Educación. Congreso de la República de Venezuela, 1980).

\subsection{Dimensión I: Legislación educativa, los niveles y los diferentes modelos de escuela}

En esta primera dimensión existen muy pocas diferencias entre países. En todos existe legislación específica nacional y niveles educativos con diferentes denominaciones, pero con muchas similitudes. Se encontró que, en todos tres niveles educativos, un primer nivel denominado Infantil (España, República Dominicana), Parvulario en Chile o Preescolar (Portugal, Colombia); un segundo bajo los nombres de Primer Nivel (Venezuela), Básico (Chile y Portugal) o Primaria (Colombia, España, República Dominicana y Venezuela), y un tercero, en el que coincide la denominación en todos los países, Secun- daria, excepto Chile, donde recibe la denominación de Enseñanza Media.

La escolarización es obligatoria variando la edad de inicio y finalización y asistiendo desde los tres años (España), cuatro (Chile, Venezuela), cinco (Colombia) y seis años (Portugal y República Dominicana), hasta los 15 (Colombia), los 16 (España), los 17 (República Dominicana) o los 18 (Chile, Portugal y Venezuela), aunque en este último país, según la modalidad de Secundaria que cursen, pueden finalizar a los 19 años.

En cuanto al modelo de escuela en el cual se organiza este proceso, predomina uno basado en público y privado (subvencionado por el Estado o no). En Chile, establecimientos municipales, particulares subvencionados y los particulares pagados; en Colombia y España se denominan públicas, concertadas (subvencionadas por el Estado) y privadas; en Portugal y Venezuela, públicas, privadas subvencionadas por el Estado y privadas, y en República Dominicana, públicos, privados y semiprivados. En el caso de Venezuela, las escuelas subvencionadas se corresponden con escuelas católicas.

En Venezuela y en República Dominicana se encontró un matiz diferenciador al existir, en el primer país, escuelas de turno integral o de medio turno, dependiendo del número de horas/día que el alumnado acude a la escuela; en el segundo, todavía existen jornadas escolares hasta medio día que comprenden aproximadamente cuatro horas y media de docencia.

\subsection{Dimensión II: Número de horas que la asignatura EF tiene a la semana}

En Chile y España el estudio encontró, dos horas en el Nivel Inicial (en Chile cuatro horas/semana de una a cuatro) y dos en el Secundario, aunque en Espańa existe la posibilidad de aumentar la carga horaria con asignaturas optativas en la enseñanza Secundaria, lo que puede aumentar la carga a tres horas semanales (esta aparición de materias optativas es decisión de cada centro educativo). En República Dominicana, se contemplan tres sesiones de 
45 minutos por semana en Primaria y dos sesiones de 45 minutos por semana en Secundaria. Por último, en Venezuela en el nivel de Primaria son 120 minutos, con una frecuencia de una vez a la semana (en los casos en que el profesorado tenga horas disponibles, podría ser dos veces por semana y en Secundaria, son 45 minutos cada sesión de clase, con una frecuencia de tres veces por semana en las escuelas de medio turno. En las de turno Integral son 90 minutos cada sesión de clase, con una frecuencia de tres veces por semana).

En Portugal se diferencia más por edades, hasta los nueve años, cinco horas a la semana dedicados a la EF y la Educación Artística (artes visuales, expresión dramática/teatro, danza y música). A partir de esa edad, 150 minutos por semana, aunque en virtud de la legislación sobre la ley de flexibilidad en la gestión del currículo, algunas escuelas pueden asignar una mayor carga a la EF (por ejemplo, 180 minutos/semana).

Hay que reseñar que en Colombia no existe una ley que defina las horas semanales de EF en la Básica Primaria y Secundaria, por lo tanto, cada institución educativa puede determinar un mínimo y un máximo de horas. En el caso de Primaria, en las instituciones públicas se puede contar con dos horas a la semana si es un colegio privado, puede tener hasta tres horas semanales. Para la Secundaria, hay colegios con dos o tres horas semanales tanto públicos como privados.

\subsection{Dimensión III: El currículo escolar}

En la tercera dimensión, al tratar el grado de prescripción y carácter del currículo (nacional/estadal/ provincial/municipal) en cuanto a la legislación de la EF, se aborda más específicamente la temática en el currículo escolar, revelándose que en Chile, España, Portugal, República Dominicana y Venezuela, el currículo está recogido en la legislación del país y es de cumplimiento obligatorio. Tiene carácter nacional, aunque pueden darse algunos aspectos regionales/ locales que permitan algunas adaptaciones (adaptar algunos elementos dependiendo de las necesidades, debilidades y fortalezas de su entorno social, geográ- fico y económico), y cada escuela tiene autonomía para cumplirlo. Vuelve a ser Colombia el único país donde el currículo no se encuentra recogido en la legislación. Desde el Ministerio de Educación Nacional de citado país se elaboran documentos específicos para la EF, Lineamientos Curriculares de la EF, Recreación y Deporte y las Orientaciones Pedagógicas para la EF, Recreación y Deporte) pero no a nivel legislativo.

La Tabla 2 se presenta los elementos didácticos que están recogidos en la legislación de los países donde se encuentra el currículo reglamentado.

En cuanto a la existencia de programas organizados de deporte escolar, se pudo comprobar que, en todos los países existen pero con diferentes estructuras, dependencias orgánicas e intencionalidades.

Las líneas básicas de los programas existentes en el ámbito del deporte escolar en cada país serían:

En Chile son instancias pre-deportivas y deportivas diseñadas por el Ministerio del Deporte (2019). El programa que está en desarrollo actualmente es el programa de deporte formativo Crecer en Movimiento, motivado por la relevancia de la actividad física y el deporte escolar en la batalla contra la obesidad infantil y el desarrollo integral de niños, niñas y adolescentes. Este programa apunta a un mayor desarrollo de las habilidades motrices de los preescolares, hacer efectiva las horas de EF integrando por primera vez a los alumnos de la Educación Media. Todas las actividades se ejecutan tres veces a la semana. El tiempo de cada sesión es de 60 minutos. Las actividades se implementan en todas las regiones y comunas del país. El programa tiene un modelo de desarrollo que comprende tres líneas de acción: (1) jugar y aprender; (2) del juego al deporte y (3) escuelas de elección deportiva.

Colombia tiene en su Sistema Nacional del Deporte el Programa Supérate Intercolegiados, el cual es un Sistema Nacional de Competencias Deportivas y Académicas que busca generar oportunidades al ejercicio del derecho al deporte y a la educación, 
Tabla 2. Elementos didácticos recogidos en la legislación del currículo de EF

\begin{tabular}{|c|c|c|c|c|c|}
\hline & Venezuela & $\begin{array}{c}\text { República Domi- } \\
\text { nicana }\end{array}$ & Chile & Portugal & España \\
\hline Competencias & $\begin{array}{l}\text { X (potencialida- } \\
\text { des) }\end{array}$ & $x$ & $x$ & $\mathrm{x}$ & $x$ \\
\hline Objetivos & X (propósitos) & & $x$ & $x$ & $x$ \\
\hline Contenidos & $X$ (referentes) & $x$ & $x$ & $x$ & $x$ \\
\hline Actividades/tareas & $x$ & & $x$ & & \\
\hline $\begin{array}{l}\text { Criterios de eva- } \\
\text { luación }\end{array}$ & $x$ & $x$ & $x$ & $x$ & $x$ \\
\hline $\begin{array}{l}\text { Resultados de } \\
\text { aprendizaje }\end{array}$ & $X$ & $x$ & $x$ & $\mathrm{x}$ & $x$ \\
\hline Métodos & $x$ & $x$ & $X$ & $\mathrm{X}$ & $x$ \\
\hline Otros & $\begin{array}{l}\text { Primaria evalúa de } \\
\text { manera cualitati- } \\
\text { va y Secundaria } \\
\text { cuantitativa }\end{array}$ & $\begin{array}{l}\text { Indicadores de } \\
\text { logro y estrategias } \\
\text { de planificación }\end{array}$ & $\begin{array}{l}\text { Propósitos, es- } \\
\text { trategias sugeri- } \\
\text { das, objetivos de } \\
\text { aprendizaje, objeti- } \\
\text { vos transversales, } \\
\text { aptitudes. }\end{array}$ & $\begin{array}{l}\text { Aprendizajes } \\
\text { esenciales por año } \\
\text { y ciclo de escola- } \\
\text { ridad }\end{array}$ & $\begin{array}{l}\text { Bloques de conte- } \\
\text { nidos }\end{array}$ \\
\hline
\end{tabular}

dirigido a niños, niñas, adolescentes y jóvenes escolarizados y no escolarizados, entre siete y 17 ańos de edad, a través de competencias y procesos de formación deportivos y actividades complementarias.

Estos juegos son organizados en cada municipio y departamento por el ente deportivo correspondiente con el apoyo del profesor de EF de las instituciones educativas. De manera alternativa, cada instituto de deportes municipal y departamental tiene programas, proyectos y estrategias para fomentar la práctica deportiva en la edad escolar (Ley General de Educación 115/1994. Congreso de la República de Colombia, 1994).

En Portugal, la Escuela de Deportes (DE) está bajo la supervisión del Ministerio de Educación (Lei de Bases do Sistema Educativo 46/1986. Assembleia da República de Portugal, 1986), con recursos financieros y apoyo logístico adecuado para el desarrollo de competiciones y eventos deportivos. La DE se entiende como el conjunto de prácticas deportivas desarrolladas como un complemento curricular y ocupación del tiempo libre, en un régimen de libertad de participación y elección, integrado en el plan de actividades de la escuela y coordinado dentro del sistema educativo (Decre-
to-Ley 95/91). Las actividades deportivas escolares son parte de la carga de trabajo de la mayoría de los maestros de EF.

La DE se basa en un sistema universal y abierto de modalidades y prácticas deportivas, organizado de manera que integre armoniosamente las dimensiones específicas de esta actividad, a saber, la enseńanza, el entrenamiento, la recreación y la competencia, y se desarrolla en los siguientes niveles de actividad: (1) nivel I: conjunto de actividades para promover la actividad física y las actividades deportivas, organizadas además del aprendizaje esencial de la disciplina de EF; (2) nivel II: entrenamiento deportivo grupal y grupal regular y actividades formales de competencia deportiva entre escuelas de alcance local, regional, nacional y posiblemente internacional; (3) nivel III: actividades para profundizar la práctica deportiva, el entrenamiento y la competencia, en modalidades y grupos con alto potencial deportivo; (4) centros de entrenamiento deportivo: centros de desarrollo deportivo, organizados por grupos escolares y escuelas no agrupadas, en asociación con federaciones, municipalidades y organizaciones locales con el objetivo de mejorar el rendimiento deportivo a través de la concentración de recursos humanos y materiales en lugares donde los estudiantes de varios grupos pue- 
den converger, ya sea durante los períodos escolares o en etapas de entrenamiento deportivo especializado, en las vacaciones escolares.

En España, no se organizan igual en todas las comunidades autónomas. En Galicia, comunidad que se utilizará como referencia en este estudio se gestiona a través del Plan Galicia Saudable, que coordina, a través del Plan Proxecta (Xunta de Galicia, 2019), diferentes programas orientados al fomento de la actividad física y el deporte en el contexto escolar. Uno de ellos es el denominado XOGADE, que es el que se corresponde con el modelo del deporte escolar. El profesor de EF suele ser el coordinador, pero pueden participar otros docentes, monitores, etc. Es una iniciativa promocionada y financiada por la Xunta de Galicia, con el objetivo de llevar la actividad a todos los ayuntamientos gallegos y brindarles a los escolares la posibilidad de practicar y competir con los clubs y colegios de toda la comunidad autónoma. Se le da así respuesta a uno de los objetivos principales de la política deportiva de la Xunta de Galicia: fomentar y extender el deporte educativo y saludable a toda la sociedad desde las edades más tempranas. Este programa va dirigido a todos los escolares gallegos, por lo que pueden inscribirse, si lo desean, los centros de enseñanza, las asociaciones de madres y padres de alumnos, clubes, escuelas deportivas municipales, agrupaciones deportivas, asociaciones, etc., pertenecientes a la Comunidad Autónoma de Galicia.

Hay que reseñar además la existencia de otros programas no dirigidos específicamente al deporte escolar, sino al fomento en general de la práctica educativa de los escolares gallegos, tales como: (1) el Proyecto Deportivo de Centro, que pretende ser el instrumento de planificación, desarrollo y evaluación de los programas de fomento de la actividad física, ejercicio físico, el deporte para la salud en los escolares gallegos, en los centros educativos; (2) DAFIS, que es una plataforma impulsada por diferentes órganos públicos, cuyo fin es que la valoración de la condición física en relación con la salud se trabaje en el ámbito escolar de un modo transversal, introduciendo las nuevas técnicas y haciendo partícipe al alumnado en dicho proceso de valoración, en el análisis de resultados y en la posterior toma de decisiones para mejorar su propia condición física; (3) Más y Mejor Actividad Física, que busca posibilitar una acción común de las materias de Ciencias Sociales/Ciencias Naturales y EF que puedan promover hábitos de vida activa y saludable a través de distintas actividades; (4) Móvete + , que promueve la actividad física a través de una acción lúdica, transversal, divertida y, al mismo tiempo, la promoción de más ejercicio a través de la coreografía, la música y la letra original, y su aplicación en las clases de EF; (5) Ir en bici/andando a la escuela, que implica el diseño e implementación de rutas seguras para caminar o andar en bicicleta y la realización de talleres de seguridad vial, y (6) la Milla Diaria, que consiste en realizar una milla diaria (andando/trotando) dentro del horario lectivo.

En la República Dominicana existen juegos deportivos nacionales escolares (Ley Orgánica de Educación de la República Dominicana 66-1997, 1997). Dirigidos por el Ministerio de Educación (MINERD), el cual realiza el calendario del certamen y monitorea su ejecución con el apoyo de su personal (técnicos de EF) y los docentes de EF de los centros educativos. Estos juegos presentan una primera orientación claramente educativa fomentando la realización de actividades deportivas en las escuelas, promoviendo el deporte y la recreación para la formación de los escolares, y una segunda dirigida al desarrollo en las escuelas de los talentos deportivos que en el futuro integren las selecciones nacionales que representarán al país en eventos internacionales. Para estas dos vertientes, el Ministerio cuenta con la colaboración del Instituto Nacional de Educación Física (órgano responsable en el área educativa) y el Comité Olímpico Dominicano (responsable en el área deportiva).

En Venezuela, es responsabilidad de la Dirección de Deporte Escolar, que coordina los Juegos Nacionales Escolares anualmente (Ley Orgánica de Educación. Congreso de la República de Venezuela, 1980). Los deportes son seleccionados a nivel nacional. En conjunto el Ministerio de Educación (Ministerio del Poder Popular para la Educación) y el Ministerio de Deporte (Ministerio del Poder Popular para la Juventud y Deportes) coordinan la actividad. Lo dirige 
el profesorado de EF de la escuela, por lo general en la misma hora de la clase de EF, en algunos casos lo hacían en horario separado. En el nuevo diseño curricular se tiene un horario denominado 'área de interés', donde los estudiantes pueden seleccionar entre artes, actividades productivas, comunitarias o deporte/recreación. En este último bloque la frecuencia de práctica es tres veces a la semana y comprende seis horas. Allí puede ser otro profesional y no afecta a la clase natural (curricular) de EF.

En Venezuela, finalmente, existe el Programa Barrio Adentro Deportivo (Organización Panamericana de la Salud, 2006) coordinado por la tríada: Deporte-Salud-Educación. Esto se desarrolla en los barrios y participan los miembros de la comunidad con actividades variadas para diferentes grupos de edades.

\subsection{Dimensión IV: Escuelas}

Atendiendo a esta nueva dimensión, con algunos matices en España y Portugal, en España y Portugal las escuelas están en su mayoría dotadas de instalaciones adecuadas y cuentan con material suficiente para el desarrollo del currículo de EF. En una situación intermedia se podría situar a Chile, pues si bien existen recursos del Estado, no se podría garantizar que el total de las escuelas están dotadas de instalaciones adecuadas ni del material suficiente para el desarrollo adecuado del currículo de EF. Por último, esto es más difícil de garantizarse en República Dominicana, aunque la situación es mejor en la pública que en la privada, ni en Venezuela y Colombia, donde se pueden observar muchas diferencias en este tema dependiendo del tipo de escuelas (público/privado) y del lugar geográfico donde se encuentren (rural/urbano), así como de las políticas de cada institución.

Respecto a la oferta de actividades extraescolares relacionadas con la actividad física en los centros educativos, una vez finalizado el horario lectivo, este estudio encontró un panorama muy parecido al anterior. Por un lado, en España, Portugal y Venezuela, en las escuelas de un solo turno, existe ese tipo de actividades de forma muy generalizada, mientras que en Chile, existen talleres extra programáticos deportivos, selec- ciones escolares y talleres psicomotores o ligados a la actividades físicas y en Colombia, los colegios privados tienen actividades extraescolares en diferentes deportes y constituyen sus propios clubes deportivos, mientras que en los colegios públicos donde hay clase de doble turno, mañana y tarde se hace más complejo) no se puede garantizar esa práctica generalizada. En República Dominicana no existe esa oferta de actividades fuera del horario lectivo, excepto en algunos centros educativos públicos denominados de "excelencia", en el que se ofrecen horas de práctica de alguna disciplina deportiva fuera del horario.

\subsection{Dimensión V: Profesorado}

Por último, la quinta dimensión hace alusión al profesorado, más específicamente, a aspectos relativos a su formación inicial y permanente y al status profesional.

En cuanto a la formación inicial, en todos los países analizados, la formación del profesorado se realiza en la universidad, con duración y estructura diversa. En Portugal y en España se necesita cursar los estudios en dos niveles de enseñanza superior, licenciatura/grado y máster; el total en ambos países es de cinco años de estudios. En el resto el nivel de estudios es el de licenciado, variando los años de duración. En Chile y Colombia, cinco años, en República Dominicana entre tres y cinco años (depende de cada universidad) y en Venezuela, actualmente, cuatro años. De la misma manera, en todos los países la formación inicial del profesorado de EF es igual a las otras áreas del conocimiento (Matemáticas, Literatura, Historia, etc.) y las universidades o el ministerio responsable organizan actividades de formación continua para el profesorado de EF, excepto en República Dominicana y Venezuela, donde pueden hacerlo, pero no es algo organizado, sino ocasional; aunque en Venezuela el Ministerio del Poder Popular para la Educación, en los últimos cuatro años, inició un plan nacional de formación y estableció una estructura para la formación permanente del profesorado, incluyendo la gratuidad de los niveles de pregrado y postgrados.

Para acceder como docente a las escuelas públicas en Colombia, España, Portugal y República Dominica- 
na, existe un sistema organizado de oposiciones y/o concurso, sin embargo, en Chile, para incorporar a un docente se debe pasar por una propuesta del equipo directivo de la unidad educativa, pero es la corporación la que al final decide a quién contrata. En Venezuela, en el pasado, era por concurso, pero en los últimos años pasó a ser por incorporación directa en instituciones del Estado en atención a las necesidades.

Con relación a derechos y deberes profesionales, excepto en República Dominicana (no en todos los ámbitos, pues los directores de centros educativos suelen priorizar las áreas de Matemáticas y Lecto-Escritura), en el resto de los países las condiciones laborales (económicas y de dedicación) del profesor de EF son iguales a las del resto de profesorado de otras asignaturas.

Finalmente, es importante destacar que en Venezuela la persona que imparte la EF es un especialista; en República Dominicana es un profesional especializado en el área, pero en algunos centros educativos no existe el profesional del área y quien imparte la materia es un docente integrador. En Chile, Colombia, España y Portugal, en los primeros ańos, es un generalista y posteriormente pasa a ser un especialista. Sin embargo, en el último país, la legislación vigente prevé la posibilidad de existencia de cooperación entre profesorado generalista y especialista, así, en edades de seis a 10 años, hay escuelas donde la EF es impartida por estos últimos.

\section{Conclusiones}

En todos los países es estudio revela que, existe legislación educativa muy similar, aunque con diferente denominación, que reglamenta la educación obligatoria, estructura los diferentes niveles educativos y regula los diferentes modelos de escuela.

La EF en el currículo nacional y el deporte escolar presenta un número de horas a la semana que varía según los niveles educativos (Primaria/Básica y Secundaria). Solo Colombia no tiene esta temática explicitada en su legislación.
En todos los países, excepto Colombia, el currículo de la EF se encuentra legislado, recogiendo elementos didácticos bastante similares en todos los países.

Aunque con matices, las escuelas están dotadas de instalaciones adecuadas y cuentan con material suficiente para el desarrollo del currículo de EF.

Este estudio encontró diferencias en cuanto a la oferta de programas organizados de deporte escolar. Varían los órganos responsables de dichos programas, los objetivos que pretenden, sus contenidos y su implicación en las escuelas.

En cuanto a la formación inicial, en los países analizados, la formación del profesorado se realiza en la universidad, con duración y estructura diversa, excepto en República Dominicana y Venezuela (aunque en esta sí de forma ocasional), y se organizan actividades de formación continua para el profesorado de EF.

Por último, en cuanto al profesorado, excepto en Chile y Venezuela, existe un sistema organizado de oposiciones y/o concurso para acceder a una plaza de profesor en la escuela pública; en general, las condiciones laborales (económicas y de dedicación) del profesor de EF son iguales a las del resto de profesorado de otras asignaturas, y en los primeros ańos la imparte un generalista y posteriormente un especialista.

\section{Referencias}

Aguilar-Farias, N., Martino-Fuentalba, P., Carcamo-Oyarzun, J., Cortinez-O’Ryan, A., Cristi-Montero, C., Von Oetinger, A., \& Sadarangani, K. P. (2018). A regional vision of physical activity, sedentary behaviour and physical education in adolescents from Latin America and the Caribbean: results from 26 countries. International Journal of Epidemiology, 47(3), 976-986.

https://doi.org/10.1093/ije/dyy033

Chin, M. K., \& Edginton, C. R. (2014). Physical Education and Health: Global Perspectives and Best Practice. Sagamore Publishing. 
Direção-Geral da Educação de Portugal (2017). Programa do Desporto Escolar 2017-2021.

https://bit.ly/36j3AM6

Klein, G., \& Hardman, K. (2008). Physical education and sport education in the European Union. Editions Revue EP.S.

Ley Orgánica de Educación. Congreso de la República de Venezuela, 28 de julio de 1980.

http://bit.ly/2rYHWxY

Lei de Bases do Sistema Educativo46/1986.Assembleia da República de Portugal, 14 de Outubro de 1986. http://bit.ly/2Rxc91E

Ley General de Educación 115/1994. Congreso de la República de Colombia, 8 de febrero de 1994. http://bit.ly/34W296j

Ley Orgánica de Educación de la República Dominicana 66-1997. Congreso Nacional de la República Dominicana, 9 de abril de 1997.

http://bit.ly/2Lvek1Y

Ley General de Educación 20370/2009. Ministerio de Educación. Gobierno de Chile, 12 de septiembre de 2009. http://bcn.cl/1uvx5

Ley No 20.529/2011. Sistema Nacional de Aseguramiento de la Calidad de la Educación Parvularia, Básica, Media y su Fiscalización. Ministerio de Educación. Gobierno de Chile, 27 de agosto de 2011. http://bcn.cl/1uv5c

McLennan, N., \& Thompson, J. (2015). Educación Física de calidad (EFC): Guia para los responsables politicos. UNESCO. https://bit.ly/2L7BUlr

Ministerio del Deporte. Gobierno de Chile (2019). Programa Crecer en Movimiento.

https://bit.ly/347tfpE

Ministerio de Educación Nacional. República de Colombia (2000). Serie lineamientos curriculares. Educación Física, Recreación y Deporte.

https://bit.ly/2LCr6Mi
Ministerio de Educación Nacional. República de Colombia (2010). Orientaciones Pedagógicas para la Educación Física, Recreación y Deporte.

https://bit.ly/2RGgJuV

Ministerio del Poder Popular para la Educación (1959). Juegos Nacionales Escolares en Venezuela. https://bit.ly/2sfg0Wz

North Western Counties Physical Education Association (2014). World-wide Survey of School Physical Education: Final Report. UNESCO.

https://bit.ly/2q20WuL

Organización Panamericana de la Salud (2006). Barrio Adentro: Derecho a la salud e inclusión social en Venezuela. Centro de Documentación e Información. OPS Venezuela. http://bit.ly/2Rq8BOO

OECD (2019). OECD Future of Education 2030. Making Physical Education Dynamic and Inclusive for 2030. International Curriculum Analysis. OECD. https://bit.ly/2pZdrXY

Pühse, U., \& Gerber, M. (Eds.) (2005). International Comparison of Physical Education: Concepts, Problems. Prospects. Meyer \& Meyer Sport.

Ribeiro, I.C., Parra, D. C., Hoehner, C. M., Soares, J., Torres, A., Pratt, M., Legetic, B., Malta, D. C., Matsudo, V., Ramos, L. R., Simoes, E. J., \&Brownson, R. C. (2010). School-based physical education programs: evidence-based physical activity interventions for youth in Latin America. Global Health Promotion 17(2), 05-15.

https://doi.org/10.1177/1757975910365231

Xunta de Galicia. Consellería de Educación, Universidade e Formación Profesional (2019). Plan Proxecta. https://bit.ly/2YuLLqZ 


\section{Cuestionario estudio comparado (Anexo 1)}

\section{Legislación de la educación en el país}
a.- Leyes de aplicación (para Educación Infantil, Inicial o Primaria y Secundaria)
b.- ¿Existe educación infantil en tu país? ¿Es obligatoria? Edades.
c.- ¿La Educación Inicial o Primaria en tu país es obligatoria? Edades.
d.- ¿La Educación Secundaria en tu país es obligatoria? Edades.
e.- ¿Qué tipos de escuelas existen: públicas, privadas, subvencionadas o concertadas?

\section{La EF en el currículo nacional y el deporte escolar}
a.- Número de horas semanales de EF en la enseñanza Primaria.
b.- Número de horas semanales de EF en la enseñanza Secundaria.

\section{La EF en el currículo escolar}

a.- ¿El currículo de la EF está recogido en la legislación el país? Si la respuesta es afirmativa:

- ¿El cumplimiento es obligatorio?

- ¿Tiene carácter nacional o cada estado/provincia/municipio puede modificarlo?

- ¿Cada escuela tiene autonomía para cumplirlo?

b.- Di qué elementos didácticos están recogidos en dicha legislación:

- Competencias.

- Objetivos.

- Contenidos.

- Actividades/tareas.

- Criterios de evaluación.

- Resultados de aprendizaje.

- Métodos.

- Otros (Di cuáles).

c.- ¿Existe un Programa de Deporte Escolar? Si la respuesta es positiva:

¡¿Cómo funciona?

- ¿Quién lo dirige? ¿El profesorado de EF de la escuela, otro profesional? 


\section{Las escuelas}
a.- ¿Están dotadas de instalaciones adecuadas?
b.- ¿Tienen material suficiente para el desarrollo del currículo de EF?
c.- ¿Hay actividades físico/deportivas propuestas por la escuela una vez finalizado el horario escolar?
d. ¿En las instalaciones de las escuelas se practican actividades físico/deportivas propuestas por clubes deportivos?
e.- ¿El horario de la clase de EF es visible en el horario escolar?
f.- ¿Puede alguien negar o ceder las horas de EF para otra área del conocimiento?

\section{El profesorado}

a.- ¿La formación del profesorado se realiza en la universidad?

b.- ¿Cuantos años de formación se requieren?

c.- ¿La formación inicial del profesorado de EF es igual a las otras áreas del conocimiento (Matemáticas, Literatura, Historia, etc.)?

d.- ¿Las universidades o el ministerio responsable organizan actividades de formación continua para profesorado de EF?

e.- ¿Para acceder como docente a las escuelas públicas qué sistema de ingreso existe? ¿Oposiciones, concurso, contratación directa por el órgano responsable?

f.- ¿Las condiciones laborales del profesor de EF son iguales a las del resto de profesorado de otras asignaturas?

g.- ¿El docente que imparte la EF es un docente especialista en el área o es un maestro integrador?

h.- ¿El que trabaja la EF en toda la Secundaria es un docente especialista en el área?

\section{CÓMO CITAR:}

González-Valeiro, M. A., Bustamante-Castaño, S. A., Chaverra-Fernández, B., Fonseca-Gomes, L. C., López-D’Amico, R., Figueira-Martins, J. F. S., Reyno-Freundt, A. M., Toja-Reboredo, B., \& Zamora-Mota, H. R. (2019). Estudio comparado: La Educación Física en Colombia, Chile, España, Portugal, República Dominicana y Venezuela. Revista Caribeña de Investigación Educativa (RECIE), 3(2), 7-18. https://doi.org/10.32541/recie.2019.v3i2.pp7-18 